\title{
Análisis de los determinantes de la predisposición a visitar un destino de turismo de bienestar: Tipologías del turista potencial
}

\section{Analysis of the determinants on the predisposition to visit a wellness tourism destination. Typologies of potential tourists}

\section{Andrea de la Hoz Correa}

Universidad de Granada, Escuela de Doctorado de Humanidades y Ciencias Sociales y Jurídicas, Campus La Cartuja, s/n, 18071, Granada, España, andrehoz@gmail.com.

\section{Francisco Muñoz Leiva}

Universidad de Granada, Departamento de Comercialización e Investigación de Mercados, Facultad de Ciencias Económicas y Empresariales, Campus La Cartuja, s/n, 18071, Granada, España, franml@ugr.es.

\section{Resumen}

En el presente artículo se analiza a) el posible efecto que variables como la familiaridad, la motivación y la imagen ejercen sobre la intención de visita de un destino de turismo de bienestar y b) la existencia de diferentes grupos de turistas utilizando criterios sicológicos, en este caso, las motivaciones. Para la obtención de datos se realizó una encuesta con cuestionario web enviado a una lista existente de inscritos en una plataforma web. El análisis evidencia la existencia de grupos diferenciados de turistas de bienestar y demuestra la influencia de las variables estudiadas sobre la intención de visitar un destino de turismo de bienestar. Los hallazgos contribuyen a un mejor entendimiento del proceso de toma de decisiones y los factores que influencian a los turistas actuales y potenciales a realizar actividades de bienestar y a elegir ciertos destinos turísticos.

Palabras claves: Turismo de bienestar, motivación, intención, efecto de la mera exposición, familiaridad, imagen de destino.

\section{Abstract}

In this article we analyze: a) the potential effect that variables such as familiarity, motivation and image have on the intention to visit a wellness tourism destination; b) the existence of different groups of tourists that may exist using a psychological criteria, in this case, motivations. For data gathering, a web questionnaire was sent to an existing entry list on a web platform. The analysis demonstrates the existence of groups of wellness tourists and shows the influence of the variables under study on the intention of visiting a wellness tourism destination. The findings contribute to a better understanding of the decision-making process and the factors that influence actual and potential tourists to conduct welfare activities and to choose certain destinations.

Keywords: Wellness tourism, motivation, intention, mere exposure effect, familiarity, destination image.

\section{Introducción}

La actividad de viajar a otros lugares en busca de un mayor bienestar físico o sicológico es un fenómeno histórico que hoy en día concentra cada vez más el interés de individuos, empresas y gobiernos por las oportunidades de generación de ingresos que ofrece. Esta industria que se denomina "turismo de salud" y ha sido valorada en US\$40 billones a nivel mundial en el 2008 y con una tasa de crecimiento potencial de un $20 \%$ por año (Morgan, 2010).

Las perspectivas apuntan a un crecimiento de la industria de salud y bienestar; sin embargo aún existen divergencias en cuanto a su definición, así como lagunas en la investigación de los diferentes tipos o subsectores como ocurre en el caso del turismo de bienestar. Tampoco se han encontrado estudios que aborden los diferentes aspectos como las motivaciones, el perfil del paciente o consumidor, agentes moderadores, etc.

En el presente artículo se cubren algunas de estas lagunas por medio del análisis del perfil de los turistas de bienestar en base a las motivaciones subyacentes y el efecto que las variables motivación, familiaridad e imagen ejercen sobre la intención de visita de este destino específico.

Con este fin se realizó una investigación empírica que mediante el diseño de un cuestionario web, obtuvo respuesta de un total de 401 turistas potenciales de un destino turístico. Los encuestados respondieron a diferentes cuestiones relativas a motivaciones, imagen, familiaridad e intención a la hora de elegir un destino, así como otras variables de clasificación.
En la siguiente sección se realiza una revisión de la literatura científica relativa a las variables de familiaridad, motivación e imagen relacionadas con el sector turístico y la justificación de una serie de hipótesis de investigación en base a los principios de la Teoría de Mera Exposición. En la sección tercera se concretan los principales aspectos metodológicos de la investigación. En la sección cuarta se realiza el análisis de datos plasmando los principales hallazgos. $\mathrm{Y}$, por último, se recogen las principales conclusiones e implicaciones así como limitaciones y futuras líneas de investigación.

\section{Revisión de la literatura}

\subsection{Conceptualización de turismo de salud y bienestar}

Es importante aclarar las definiciones relacionadas con turismo de salud, turismo de bienestar y turismo médico. El turismo de salud, representa las actividades de los consumidores que viajan a otros países por una variedad de servicios de salud (Loh, 2014) estando al mismo tiempo de vacaciones; por tanto el lugar donde se están quedando no es el lugar de residencia habitual y permanente. Según Pearcy y Lester (2012) este tipo de turismo se caracteriza por tener tres elementos; el estar lejos de casa, la salud como motivo principal y que ocurre en una instalación especializada en este tipo de actividades.

Algunos autores como Loh (2014) o Mueller y Kaufmann (2001) diferencian entre aquellos que viajan para buscar servicios que les permitan mantener su bienestar y aquellos que buscan tratamientos debido a una condición médica específica. El primero se podría denominar turismo de bienestar y el segundo turismo médico. 
En ese sentido, el turismo de bienestar se puede identificar como una subcategoría del turismo de salud porque es realizada por personas "sanas" quienes tienen como interés primordial preservar o promover su salud. Ravichandran y Suresh (2010) lo definen como la "prestación de servicios holísticos cuyo objetivo principal es la prevención de enfermedades" (p.205). Este tipo de turistas ponen un énfasis muy fuerte en cambiar su estilo de vida para ganar un alto nivel de satisfacción a través de la autorrealización (Puczkó \& Bachvarov, 2006).

\subsection{El "Efecto de la Mera Exposición" en turismo}

La teoría del Efecto de la Mera Exposición afirma que la exposición de un individuo repetidamente a un estímulo es una condición suficiente para la mejora de su actitud hacia éste (Zajonc, 1968). Por mera exposición se entiende una condición que hace que el estímulo dado sea accesible para la percepción del individuo. Incluso se ha encontrado evidencia de que el estímulo que se percibe inconscientemente puede producir efectos más grandes que los estímulos que son recibidos conscientemente (Bornstein \& D’Agostino, 1992).

Tal como se explica en la teoría y se muestra en la Figura 1, la exposición a un estímulo que en este caso es la información sobre viajes a turistas aumenta su familiaridad con el destino y genera una imagen positiva en la mayoría de los casos aumentando así la probabilidad de visita. Esta exposición repetida es lo que permitiría que las personas incrementen sus respuestas afectivas $y$ evaluativas, efecto que en sicología social se denomina "principio de familiaridad".

Figura 1 - Teoría de la Mera Exposición

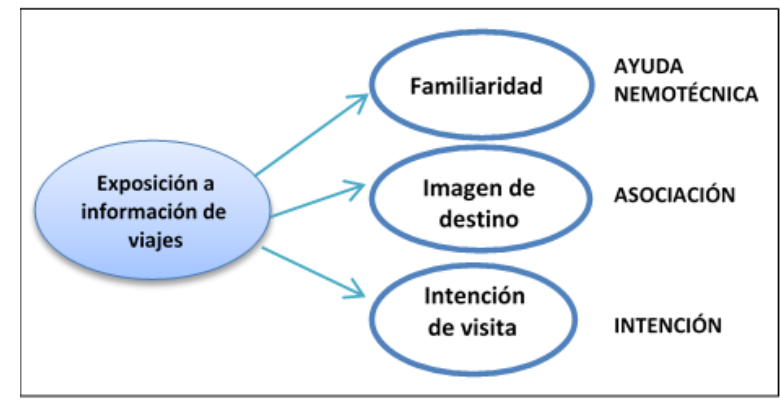

Fuente: Elaboración propia a partir de Zajonc (1968) y Bettman (1979).

Desde la publicación del trabajo de Zajonc (1968) haciendo alusión a este efecto, son muchos los trabajos que lo han utilizado para investigar relaciones en un rango amplio de fenómenos incluyendo los efectos de anuncios publicitarios, la formación de estereotipos y prejuicios, formación de actitudes, entre otros. Sin embargo muy pocos trabajos se han aplicado al área de turismo y mucho menos en turismo de bienestar. Su uso más frecuente en este campo ha sido para probar la relación que existe entre la publicidad y la imagen de un destino turístico.

Algunos ejemplos de su aplicación en turismo los podemos encontrar en el estudio de Bojanic (1991). Este estudio examina las actitudes de dos grupos de viajeros hacia un país del sur de Europa basándose en la cantidad de publicidad a la que habían sido expuestos y encuentra que el grupo expuesto a publicidad tenía actitudes más favorables sobre el destino y un mayor interés en visitarlo en un futuro cercano. Así mismo, el trabajo de Jeong
(2009) aplica la teoría de la mera exposición al analizar el efecto de la exposición a fuentes de información de viajes sobre Florida, encontrando que hay una relación directa entre la exposición, la evolución de la imagen del destino y la intención de visitarlo.

Adicionalmente en el trabajo de Jeong, Holland, Jun, y Gibson (2012) se examina el efecto de la información de una página web en la imagen de un destino revelando que la exposición a esta información afecta significativamente la imagen cognitiva y total y comprobando el efecto positivo de la mera exposición en la imagen.

\subsection{Imagen, familiaridad e intención de visita como elementos de la Teoría de la Mera exposición}

La imagen de destino se define como una representación mental, o la impresión (Bojanic, 1991; Jeong, 2009) que una persona tiene de un producto, una organización, ciudad o un país (LuqueMartínez, García, Ibañez-Zapata \& Rodríguez-Molina, 2004); en donde no reside.

Se denomina un constructo dinámico (Martínez, 2014) porque puede desarrollarse sin haber tenido contacto alguno y va modificándose a medida que aumenta la experiencia. Adicionalmente, influyen en la imagen tanto factores derivados del entorno como del propio individuo que hace la evaluación (Moreno-Gil, Beerli-Palacio \& Ledesma, 2012).

Dentro de los factores internos podemos encontrar factores sicológicos (motivación, autoconcepto, autoimagen), factores sociales (relacionados con la edad, ocupación, etc) y factores estímulo en donde se encuentran las fuentes de información y la experiencia relacionada con el destino (Baloglu \& McCleary, 1999).

Muchos estudios han encontrado que comprender cómo se forma la imagen es vital para entender como un turista realiza el proceso de selección de un destino (Beerli \& Martín, 2004; Hanlan \& Kelly, 2005; Jeong, 2009; Byon \& Zhang, 2010), pues la imagen al afectar la percepción influirá en el comportamiento subsecuente del individuo.

Por otro lado, la familiaridad se define como "las estructuras cognitivas de conocimiento relacionadas con un producto que están almacenadas en la memoria" (Marks \& Olson, 1981, p.145) o "un punto en un rango continuo de ser meramente consciente de la existencia de un producto a un estado de estar íntimamente familiarizado con él" (Jeong, 2009, p.21). De la anterior definición se desprende que bajos niveles de familiaridad pueden denominarse "conciencia" mientras que altos niveles de familiaridad pueden llamarse "conocimiento" de un producto o servicio.

La familiaridad se puede formar de experiencias directas (ej. compra previa) o experiencias indirectas (ej. exposición a publicidad, boca-oído o búsqueda de información). Por tanto varios autores defienden que esta variable está íntimamente relacionada con la adquisición de información y que las fuentes de información de viajes tienen un impacto en ella (Johnson \& Russo, 1984). 
Adicionalmente, la intención de visita se conceptualiza como el plan potencial de visitar un destino o como un tipo de inclinación a comportarse que puede incluir las acciones de "recomendar, comprar, viajar, visitar o regresar a algún lugar" y que "está determinada por una combinación de evaluaciones cognitivas y afectivas, fuentes de información usadas y motivaciones del viaje" (Jeong, 2009, p.27).

La intención y el comportamiento real están íntimamente relacionados. En realidad, muchos modelos de comportamiento del consumidor que se han construido sobre la base de la teoría de la acción razonada (TRA) enfatizan la intención como un antecedente inmediato del comportamiento real (Baloglu, 2000). A su vez numerosos estudios han demostrado, como se menciona anteriormente que las fuentes de información, motivaciones socio - sicológicas, algunas características demográficas y la imagen o actitud hacia un destino influencian las preferencias e intenciones de los turistas o consumidores (Mansfeld, 1992; Echtner \& Ritchie, 1993; Baloglu \& McCleary, 1999; Hanlan \& Kelly, 2005).

\subsection{Perfiles de turistas}

Una revisión de la literatura científica revela diferentes estudios en la extracción de grupos de consumidores de usuarios de servicios offline (Rajshekhar \& Dion, 1999; Machauer \& Morgner, 2001; Soper, 2002; Karjalouto, Mattila \& Pento, 2002; Garland, 2005; Kaynak \& Harcar, 2005; Lee, Kwon \& Schumann, 2005; Cameron, Cornish \& Nelson, 2006) y usuarios de servicios online (e.g. Schwaiger \& Locarek-Junge, 1998 [basándose teóricamente en el grado en el cual una innovación es adoptada]; Durkin, 2004 [proceso de toma de decisiones del consumidor]; Pons, 2007 [ en el contexto de la web 2.0 y el turismo rural].

Específicamente en turismo, el trabajo de Smith y Puczkó (2009) enfocado en turismo de salud concluye que la práctica de esta modalidad en Europa y Estados Unidos está dominada por personas mayores de 30 , sobre todo mujeres y su principal motivación es la "búsqueda activa de la salud" que asumen como responsabilidad propia. Adicionalmente son personas que no temen experimentar nuevos tratamientos y actividades con tal de prevenir dolencias.

La revisión sin embargo, no logra encontrar una clasificación clara en el campo del turismo de bienestar; aspecto que será cubierto con esta investigación. Así que los resultados son especialmente útiles para las diferentes compañías del sector.

\subsection{Justificación de las hipótesis de Investigación}

Muchos autores de diferentes disciplinas y campos han citado repetidamente a las variables familiaridad, la imagen cognitiva y afectiva y la motivación como determinantes en la elección de un destino antes de visitarlo (Crompton, 1979; Mansfeld, 1992; Gartner, 1993; Vogt \& Fesenmaier, 1998; Baloglu, 2000).

La teoría del "Efecto de la mera exposición" de Zajonc (1968) permite justificar las relaciones entre familiaridad, imagen e intención. La teoría dice que la exposición a factores estímulos influencia positivamente la familiaridad con un destino (Baloglu, 2000) y la imagen del destino (Baloglu \& McCleary, 1999; Beerli \& Martín, 2004; Letho, Brown, Chen \& Tsai, 2007) y, por lo tanto, repercute en mayores intenciones comportamentales de visita, compra, recomendación, etc.

La relación entre estos conceptos también puede ser explicada por el "proceso de control de la memoria" de Bettman (1979) que expresa que los sujetos en estudios verbales de aprendizaje usan estrategias mnemotécnicas, asociaciones, imágenes entre otras para la codificación de entradas recibidas y facilitar el proceso de memorización. Estas estrategias de memorización pueden ser expresadas conceptualmente como familiaridad (ej. ayudas mnemotécnicas) e imágenes percibidas (ej. imágenes o asociación). En otras palabras los turistas potenciales perciben la familiaridad con un destino y la imagen del destino como herramientas para memorizar información externa.

Por otro lado, Baloglu (2000) considera que las motivaciones afectan a la imagen de destino. Existen muchos estudios empíricos que apoyan esta relación, sin embargo también existe controversia sobre si afectan el componente cognitivo o afectivo de la imagen. Otros estudios también han demostrado que variables como las motivaciones socio-sicológicas determinan la intención de visita y comportamiento (Baloglu, 2000; Yoon \& Uysal, 2005). Específicamente que la motivación tiene un efecto positivo en la intención o afecta la intención para usar algún producto o servicio en particular (Jang \& Feng, 2007; Alegre \& Cladera, 2009).

Con base en los trabajos anteriores se plantea la siguiente hipótesis de investigación:

H1: La motivación influye positivamente sobre la intención de visitar un destino de turismo de bienestar

También se ha ilustrado que la imagen de destino influye en el propio comportamiento del turista (Pearce 1982), es decir, que destinos con imágenes positivas es más probable que sean considerados en el proceso de decisión de viajes (Mechinda, Serirat \& Gulid, 2009). En este sentido, trabajos como el de Hunt (1975) y Bigné, Sánchez y Sánchez (2001), entre otros, sostienen que en la medida en que los destinos tengan imágenes más fuertes y positivas tendrán una mayor probabilidad de ser considerados y elegidos en el proceso de decisión del viaje turístico. En otras palabras, que hay una correlación positiva entre la imagen y la intención de visita.

Además, un alto número de investigadores como Gartner (1993); Baloglu (2000) y Jeong (2009), entre otros, afirman que la imagen está formada por dos componentes, el cognitivo y afectivo. El componente cognitivo se refiere a creencias y conocimientos sobre los atributos de destino y está afectada por el conocimiento e información objetiva mientras que la evaluación afectiva se refiere a sentimientos sobre el destino y está afectada por los valores del individuo (Gartner, 1993; Baloglu \& McCleary, 1999; Martín \& Bosque, 2008).

A partir de esta información se formula la segunda y tercera hipótesis:

H2: La imagen cognitiva influye positivamente sobre la intención de visitar un destino de turismo de bienestar

H3: La imagen afectiva influye positivamente sobre la intención de visitar un destino de turismo de bienestar 
Con respecto a la familiaridad, se encuentra que esta variable influye en las percepciones del turista y el atractivo de un lugar (Mechinda, Serirat \& Gulid, 2009). Si se es familiar se percibe el lugar de manera diferente, se siente diferente y se desarrolla una imagen de ese destino (Hammitt, Backlund \& Bixler, 2006). Esta percepción puede influir en la elección de destino de un turista (Chen, 1997). Por ejemplo Milman y Pizam (1995) encuentran que la familiaridad tiene un impacto positivo en el interés y la probabilidad de visita. Esto apoya la cuarta hipótesis de investigación:

H4: La familiaridad influye positivamente sobre la intención de visitar un destino de turismo de bienestar

En el modelo de comportamiento propuesto (Figura 2) aparecen recogidas estas hipótesis de investigación que serán contrastadas en el apartado de análisis de datos.

Figura 2 - Modelo de comportamiento propuesto

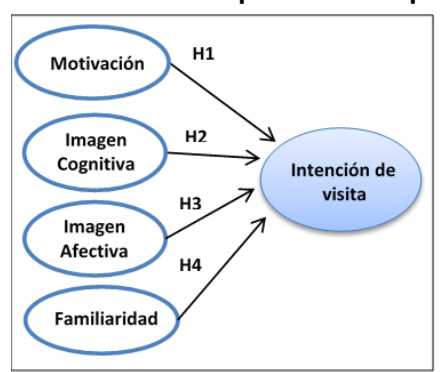

Fuente: Elaboración propia a partir de Zajonc (1968).

\section{Planteamiento metodológico}

\subsection{Proceso de recogida de datos}

La recogida de datos de la investigación se lleva a cabo entre el 26 de junio y el 25 de julio de 2013. La información relevante se resume en la Tabla 1. Se realiza una encuesta web invitando a un total de 4.000 estudiantes y egresados de una universidad del sur de España. La población de estudio estuvo constituida por turistas potenciales que buscan bienestar. La participación fue voluntaria y se ofreció un incentivo para las personas que contestaran la encuesta correspondiente a un bono Smartbox "Spa para Dos" personas. El tamaño muestral final fue de 401 cuestionarios, lo que supone un error muestral de 4,9\% para un nivel de confianza del $95 \%$. La tasa de respuesta fue del $10,4 \%$ tras el envío masivo de invitación.
Tabla 1 - Ficha técnica del estudio

\begin{tabular}{|l|l|}
\hline \multicolumn{1}{|c|}{ Población } & $\begin{array}{c}\text { Población española de turistas que realizan } \\
\text { turismo de bienestar }\end{array}$ \\
\hline Marco Muestral & $\begin{array}{l}\text { Listado de estudiantes inscritos en distintas } \\
\text { plataformas web de la universidad. } \\
\mathrm{n}=401 \text { casos válidos }\end{array}$ \\
\hline Muestra & Muestreo por conveniencia \\
\hline Tipo de Muestreo & Del 26 de junio al 25 de Julio de 2012 \\
\hline Trabajo de campo & $\begin{array}{l}\mathbf{4}, 9 \% \text { (para la estimación de una proporción, } \\
\text { donde } \mathrm{P}=\mathrm{Q}=0,5 \text { y un nivel de confianza del } 95 \%)\end{array}$ \\
\hline Error muestral
\end{tabular}

Se escogió la provincia de Granada como destino pues se caracteriza por su oferta de aguas mineromedicinales para el tratamiento de enfermedades, así como para el descanso y esparcimiento personal (Henn, Lopes, Goncalves, \& Fraiz, 2008) constituyéndose en un destino de bienestar apetecido por los turistas tanto del propio país como del resto del mundo. Actualmente ocupa la cuarta posición como destino de turismo de salud en España, tras las comunidades de Galicia, Cataluña y Aragón (Larrubia -Vargas \& Luque-Gil, 2002).

Se utilizó un muestreo por conveniencia a estudiantes en vez de personas de mayor edad porque eran sujetos accesibles y estaban motivados a participar. Trabajos anteriores como los de Reddy, York y Brannon (2010) o Phau, Shanka y Dhayan (2010) utilizan muestras de estudiantes dada la naturaleza preliminar de los estudios en este campo del turismo.

Una ventaja de usar muestras de estudiantes es que permite un grado razonable de homogeneidad entre todos los participantes, reduciendo el número de variables fuera del modelo que puedan contaminar diferencias comparativas (Winsted, 1997). Adicionalmente se considera que los jóvenes y estudiantes son un segmento cada vez más reconocido y lucrativo en el mercado mundial de los viajes.

La composición sociodemográfica de la muestra y el marco muestral se detallan en la Tabla 2 y Tabla 3. La muestra está conformada en su mayoría por mujeres $(64,8 \%)$ y jóvenes menores de 34 años $(97,3 \%)$ que están realizando sus estudios universitarios (83,3\%). En cuanto a su estado civil, el 78\%,4 son personas solteras y el nivel de ingresos en el hogar mayoritariamente es de 601 a 1200 euros (35,5\%).

Tabla 2 - Perfil sociodemográfico de la muestra

\begin{tabular}{|l|l|l|l|}
\hline \multicolumn{5}{|c}{ Características de la muestra (\%) N(401 - 318*) } \\
\hline Género & & Educación & \\
\hline Mujer & $64,80 \%$ & Posgrado & $11,00 \%$ \\
\hline Hombre & $35,20 \%$ & Secundaria(BUP/FP/Bachiller) & $5,70 \%$ \\
\hline & & Universitarios & $83,30 \%$ \\
\hline Edad & & Estado Civil & \\
\hline $18-24$ años & $56,40 \%$ & Otras situaciones & $2,30 \%$ \\
\hline $25-34$ años & $40,90 \%$ & Parejas con hijos a su cargo & $2,50 \%$ \\
\hline $35-44$ años & $2,50 \%$ & Parejas sin hijos a su cargo & $16,60 \%$ \\
\hline $55-64$ años & $0,20 \%$ & Soltero/a & $78,40 \%$ \\
\hline & & Viudo/a & $0,30 \%$ \\
\hline
\end{tabular}




\begin{tabular}{|l|l|l|l|}
\hline \multicolumn{4}{|c|}{ Características de la muestra (\%) N(401 - 318*) } \\
\hline Ocupación & & Ingresos & \\
\hline Amo/a de casa & $0,20 \%$ & Hasta 600 euros & $16,40 \%$ \\
\hline Desempleado & $10,00 \%$ & De 601 a 1200 euros & $35,50 \%$ \\
\hline Estudiante & $62,80 \%$ & de 1201 a 1800 euros & $26,10 \%$ \\
\hline Jubilado & $0,20 \%$ & Más de 1801 euros & $22,00 \%$ \\
\hline Trabajador a tiempo completo & $20,00 \%$ & & \\
\hline Trabajador tiempo parcial & $6,70 \%$ & & \\
\hline *En algunos casos hay missing data & & &
\end{tabular}

Tabla 3 - Procedencia del marco muestral

\begin{tabular}{|l|c|}
\hline \multicolumn{1}{|c|}{ Comunidad autónoma } & \% por comunidad \\
\hline Andalucía & $68,6 \%$ \\
\hline Aragón & $0,2 \%$ \\
\hline Cantabria & $0,1 \%$ \\
\hline Castilla y León & $0,4 \%$ \\
\hline Castilla-La Mancha & $1,2 \%$ \\
\hline Cataluña & $1,6 \%$ \\
\hline Ceuta (Ciudad Autónoma) & $1,7 \%$ \\
\hline Comunidad de Madrid & $6,5 \%$ \\
\hline Comunidad Valenciana & $1,5 \%$ \\
\hline Extremadura & $0,6 \%$ \\
\hline Galicia & $0,2 \%$ \\
\hline Islas Baleares & $1,1 \%$ \\
\hline Islas Canarias & $1,6 \%$ \\
\hline La Rioja & $0,1 \%$ \\
\hline Melilla (Ciudad Autónoma) & $2,0 \%$ \\
\hline Navarra & $0,2 \%$ \\
\hline País Vasco & $0,5 \%$ \\
\hline Principado de Asturias & $0,1 \%$ \\
\hline Región de Murcia & $1,7 \%$ \\
\hline Extranjero & $10,0 \%$ \\
\hline NS/NC & $0,2 \%$ \\
\hline Total & $100 \%$ \\
\hline
\end{tabular}

Fuente: Universidad de Granada (2011).

\subsection{Escalas de medida utilizadas y fiabilidad de las escalas}

Para la elaboración del cuestionario se utilizaron una serie de preguntas referentes a las características demográficas de los turistas como sexo, nivel de estudios, situación familiar, edad, situación de empleo y nivel de ingresos en el hogar. Así mismo, en la Tabla 4 se muestran las escalas multi-ítem desarrolladas a partir de trabajos científicos anteriores con las cuales se midieron la motivación, familiaridad e imagen cognitiva y afectiva, e intención de visita de un turismo de bienestar.

Tabla 4 - Ítems en cada una de las escalas de medida

\begin{tabular}{|c|c|}
\hline Imagen Cognitiva & Motivación \\
\hline $\begin{array}{l}\text { - } \quad \text { Tiene muchos lugares interesantes que visitar } \\
\text { - } \quad \text { Tiene actividades de relajación, yoga, fitness. } \\
\text { - } \quad \text { Ofrece una gran variedad de actividades al aire libre } \\
\text { - } \quad \text { Ofrece opciones adicionales de diversión } \\
\text { - } \quad \text { Tiene un lugar seguro para visitar } \\
\text { - } \quad \text { Tiene un buen número de spas y hoteles de calidad } \\
\text { - } \quad \text { Ofrece un buen número de atracciones naturales hermosas y } \\
\text { - } \quad \text { adecuadas para realizar turismo de bienestar } \\
\text { - } \quad \text { Tiene un buen clima } \\
\text { - } \quad \text { Tiene muchos festivales locales interesantes } \\
\text { - } \quad \text { Tienen suficientes sitios históricos y culturales } \\
\text { - } \quad \text { Es un buen lugar para aumentar el conocimiento }\end{array}$ & $\begin{array}{l}\text { - } \quad \text { Conciencia por la salud } \\
\text { - } \quad \text { Realizar terapia física } \\
\text { - } \quad \text { Realizar una actividad social } \\
\text { - } \quad \text { Mejora de la calidad de vida } \\
\text { - } \quad \text { Realizar una actividad que no requiere esfuerzo } \\
\text { - } \quad \text { Recomendación de otros (Comentarios de amigos y conocidos) } \\
\text { - } \quad \text { Relajación } \\
\text { - } \quad \text { Curiosidad } \\
\text { - } \quad \text { Realizar terapia mental } \\
\text { - } \quad \text { Disfrute de la naturaleza } \\
\text { - } \quad \text { Meditación } \\
\text { - } \quad \text { Vínculo con actividades comerciales }\end{array}$ \\
\hline
\end{tabular}




\begin{tabular}{|c|}
\hline Imagen Afectiva \\
\hline $\begin{array}{ll}\text { - } & \text { Desagradable/Agradable } \\
\text { - } & \text { Aburrida/Excitante } \\
\text { - } & \text { Eranquilizante/Estimulante } \\
\text { - } & \text { Arriesgada/Relajante } \\
\text { - } & \text { Poco atractiva/atractiva } \\
\text { - } & \text { Caómodica/Estable } \\
& \text { Simple/Diversa }\end{array}$ \\
\hline Fuentes de Información \\
\hline 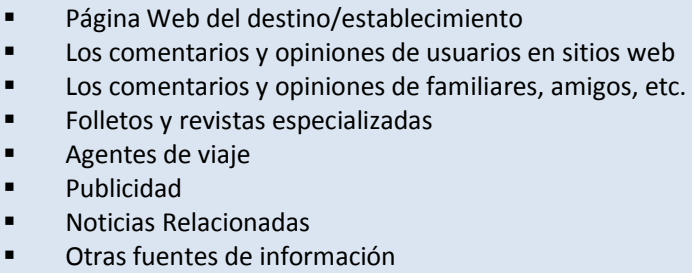 \\
\hline
\end{tabular}

Para el constructo de familiaridad (compuesto por 3 ítems) se utilizó una escala de medida tipo Likert con cinco puntos (donde 1 indica "nada familiar" y $5=$ "muy familiar") adaptada de la literatura relacionada con la temática bajo estudio, concretamente de los trabajos de Kent y Allen (1994) y Jeong (2009). Las escalas de imagen cognitiva (compuesta por 13 ítems) e imagen afectiva (compuesta por 9 ítems) se adaptaron de los trabajos de Baloglu y Mangaloglu (2001); Baloglu y McCleary (1999); Beerli y Martín (2004) con una escala de cinco puntos ( $1=$ "totalmente en desacuerdo" y 5 = "totalmente de acuerdo").

Para el constructo de motivación (compuesta por 16 ítems) se utilizó también una escala de cinco puntos ( 1 = "totalmente en desacuerdo" y 5 = "totalmente de acuerdo") adaptada del trabajo de Chen, Prebensen y Huan (2008). Debido a la escasez de trabajos sobre motivación en turismo de bienestar no se pudo utilizar una escala ampliamente difundida y validada en este caso.

Para la intención comportamental (compuesta por 5 ítems) se adaptaron escalas de varios estudios como los de Baloglu y McCleary (1999); Lam y Hsu (2006); Chen y Tsai (2007) y Chen et al. (2008). Se utilizó también una escala de cinco puntos (1 = "Muy improbable" y 5 = "Muy probable").

Una serie de análisis exploratorios fueron ejecutados para comprobar la fiabilidad de las escalas de medida. En primer lugar se utilizó el indicador alpha de Cronbach considerando el 0,7 como valor de referencia para que una escala sea considerada fiable (Nunnally, 1978). Se comprobó de esta forma que todas las variables del estudio contienen valores superiores al mínimo requerido (ver tabla 5). Por tanto se puede decir que los ítems explican gran parte de la varianza de los constructos y se comprueba su elevada consistencia interna.

Tabla 5 - Fiabilidad de las escalas

\begin{tabular}{|l|c|c|}
\hline \multicolumn{1}{|c|}{ Variable } & Elementos & Alpha de Cronbach \\
\hline Familiaridad & 3 & 0,768 \\
\hline Motivación & 16 & 0,775 \\
\hline Imagen Cognitiva & 13 & 0,818 \\
\hline Imagen afectiva & 9 & 0,756 \\
\hline Intención de visita & 5 & 0,745 \\
\hline
\end{tabular}

\section{Familiaridad}

- He escuchado hablar de las actividades y los destinos en los que se puede realizar turismo de bienestar

- Estoy familiarizado/a con el término "turismo de bienestar"

- Estoy familiarizado/a con la provincia de Granada como destino de turismo de bienestar

\section{Intención Comportamental}

- Recomendaría la Provincia de Granada como lugar de turismo de bienestar a otras personas

- La Provincia de Granada está entre mis opciones de destino en los próximos 3 años

- Evaluaría la posibilidad de ir a lugares similares en mis vacaciones en los próximos 3 años

- La Provincia de Granada está entre mis opciones de destino para las vacaciones en algún momento de mi vida

- Establecimientos centrados en turismo de bienestar están entre mis opciones de destino para futuras vacaciones

\subsection{Diseño del modelo y justificación de las herramientas de análisis de datos}

\subsubsection{Análisis Clúster}

La segmentación de mercados ha sido ampliamente utilizada en la investigación en viajes y turismo y ha recibido amplia aprobación por parte de académicos y profesionales (Frochot \& Morrison, 2000).

Esta metodología está basada en los diferentes beneficios que los consumidores buscan en un producto o servicio (principalmente beneficios utilitarios, beneficios racionales $y$ beneficios emocionales) y refleja las características específicas del destino o servicio, en lugar de sólo agrupar a los consumidores por factores tradicionales como los demográficos.

Diversas técnicas de análisis de datos, tales como análisis de conglomerados (principalmente el método de partición de kmedias) se han convertido en una forma muy popular de identificar segmentos de mercado basándose en datos recolectados a través de encuestas (Frochot \& Morrison, 2000; Dolnicar, 2002).

Dolnicar (2002) ilustra cómo suelen realizarse estudios de segmentación basadas en datos en el campo de la investigación de viajes y turismo. Por otro lado en el trabajo de Mueller y Kaufmann (2001) enfocado en turismo de bienestar, se utiliza el análisis clúster para segmentar a los huéspedes de diversos hoteles con el fin de lograr una mejor comprensión del mercado midiendo la importancia de los componentes de bienestar y los parámetros utilizados por los consumidores para su estadía en el hotel.

Sin embargo las definiciones metodologías y definiciones utilizadas hasta el momento varían en gran medida (Frochot \& Morrison, 2000). Muy pocos estudios han segmentado turistas según su comportamiento o conducta antes de decidirse a hacer un viaje. En el presente estudio se analiza este comportamiento junto con variables de clasificación.

\subsubsection{Diseño del modelo lineal propuesto: regresión lineal}

Para analizar los efectos de los determinantes (motivación, imagen afectiva y cognitiva y familiaridad) de la intención comportamental 
se realizó una regresión lineal múltiple. El modelo propuesto a ser contrastado adopta la siguiente forma:

$Y_{i}=\beta_{0}+\beta_{1} \cdot X_{i 1}+\beta_{2} \cdot X_{i 2}+\beta_{3} \cdot X_{i 3}+\ldots+\beta_{4} \cdot X_{i 4}+\varepsilon_{i}$

Donde,

$Y_{i}=$ Valor de la variable dependiente para la observación i-ésima (intención)

$X_{i j}=$ Valor de la variable independiente (familiaridad, motivación, imagen cognitiva y afectiva)

\section{$\beta_{0}=$ Ordenada en el origen o término independiente}

$\beta_{j}=$ Parámetros o coeficientes del modelo que relacionan a cada $X_{j}$ con $Y_{i}$. Representan la derivada parcial de $Y$ respecto de cada una de las $X_{j}$.

\section{$\varepsilon_{i}=$ Término de perturbación aleatoria}

De igual manera se verifica el cumplimiento de los supuestos básicos en regresión lineal tales como un número de casos suficiente, ausencia de multicolinealidad, homocedasticidad en el comportamiento de las perturbaciones, autocorrelación de las perturbaciones y la normalidad de la variable dependiente (Chica \& Frías, 2000).

Por último, como medida de la capacidad explicativa del modelo extraído se examinó el valor $R$ cuadrado $\left(R^{2}\right)$, que representa el porcentaje de varianza explicada de la variable dependiente o la proporción de las variaciones de $Y$ que son explicadas por las independientes (Chica \& Frías, 2000).

\section{Hallazgos}

\subsection{Agrupación del turista potencial}

Con el fin de conocer cuál es el perfil característico de los turistas considerando las motivaciones subyacentes a la realización de turismo de bienestar, se realizó un análisis clúster. Para identificar el número apropiado de grupos primero se procedió a realizar un procedimiento jerárquico bajo el método de Ward y la utilización media de la distancia euclídea al cuadrado. Utilizando el dendrograma y como criterio de selección el $25 \%$ de la distancia máxima de unión (Luque-Martínez, 2000) se optó por elegir cuatro grupos. Se eligió esta solución como la más viable en términos de identificación de los entrevistados e interpretación de los resultados.

A continuación se procedió a interpretar las puntuaciones medias de cada motivación y grupo (Ver figura 3). También se realizó una tabulación cruzada para examinar las diferencias entre los grupos para cada característica socio-demográfica, realización o no de turismo de bienestar, intención y familiaridad. Sólo las diferencias que resultaron ser estadísticamente significativas a un nivel del 0,05 se mencionan en el perfil. La descripción y nombre de cada uno de los grupos se presenta a continuación.

Figura 3 - Gráfico de medias

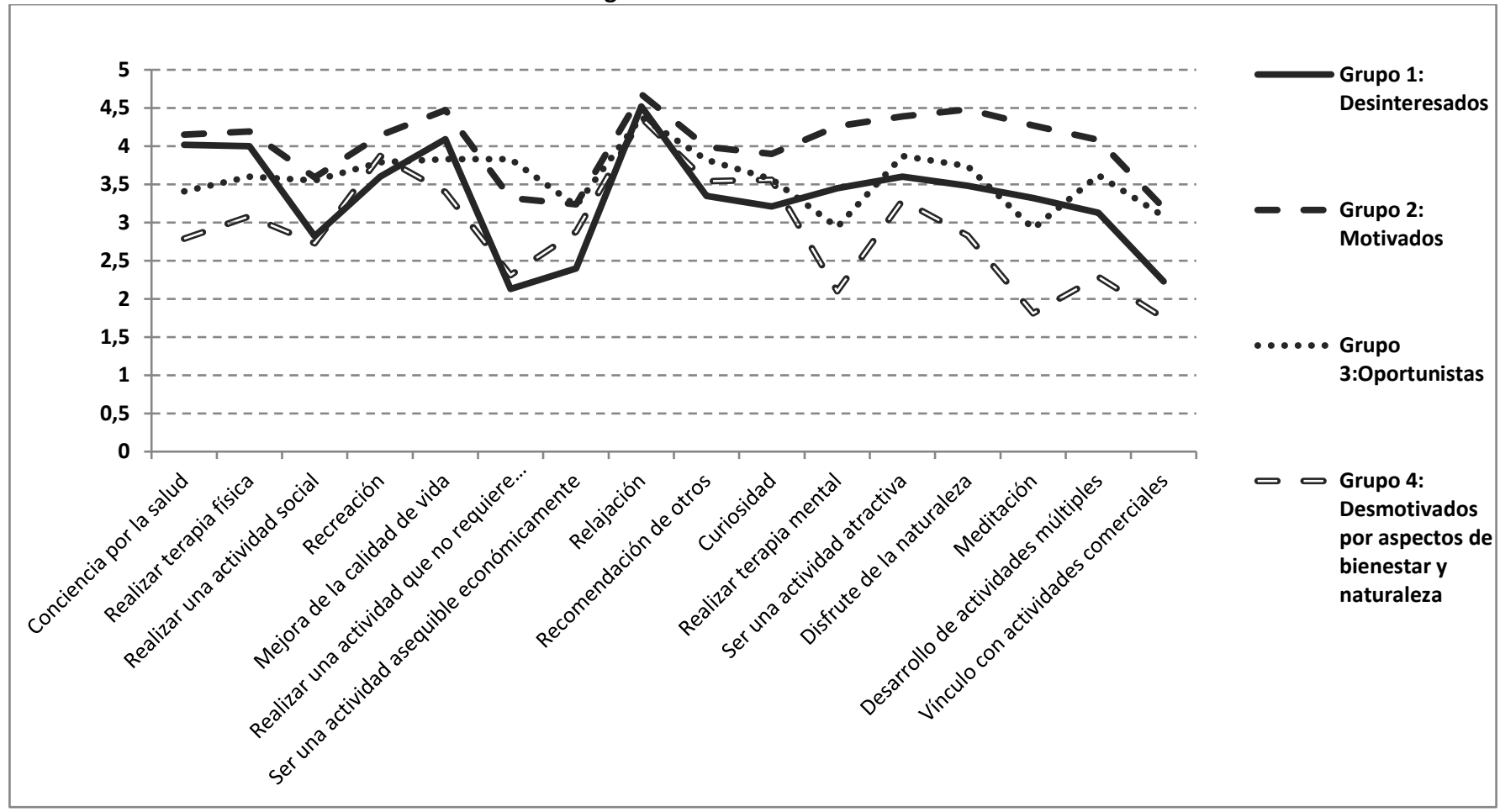

Fuente: Elaboración propia.

Grupo 1: El primer clúster representa el $23,8 \%$ de la muestra (96 integrantes). Este clúster se denomina "Desinteresados" por indicar una falta de interés entre sus integrantes por la mayoría de los factores motivacionales externos tales como la recreación, accesibilidad económica de las actividades de bienestar o el vínculo con otras actividades. También son los menos interesados en realizar actividades de bienestar porque otros lo recomienden o porque sientan curiosidad por este tipo de turismo.
Su principal motivación es la relajación y lo que menos les motiva es que sea una actividad que requiera esfuerzo físico. Los ítems "Conciencia por la salud", "Realizar terapia física", "Mejora de la calidad de vida", "Realizar terapia mental" y "Meditación" fueron los factores motivacionales con la segunda media más alta de los cuatro grupos lo que puede indicar que los integrantes de este grupo prefieren realizar turismo de bienestar por mantenerse en buena forma pero que no implique gran esfuerzo. Está compuesto 
en su mayor parte por personas cuyos hogares cuentan con ingresos entre 1201 a 1800 euros (37\%) seguido de las personas con ingresos en los hogares de 601 a 1200 euros (31,5\%). El 54,2\% de sus integrantes no han realizado turismo de bienestar siendo el porcentaje más alto tanto dentro del grupo como de la muestra. Tampoco se encuentran familiarizados con el término "bienestar" en su mayoría (31\%) y se encuentran "poco familiarizados" con la provincia de Granada como un destino de bienestar (29,8\%). Esto puede explicar en parte su falta de interés por este tipo de turismo.

Con respecto a su intención futura para una tercera parte aproximadamente (33\%) no es ni probable ni improbable que la provincia de Granada esté entre sus opciones de vacaciones en un futuro cercano, pero sí es muy probable que lo sea en algún momento de su vida $(37,5 \%)$ y también es probable que visiten establecimientos relacionados con turismo de bienestar en general en futuras vacaciones $(42,7 \%)$.

Grupo 2: El segundo clúster representa el 35,48\% de la muestra (143 integrantes) y es el segmento más numeroso. Podemos denominar a este grupo como los "Motivados" al registrar una alta motivación a la hora de realizar turismo de bienestar. Para casi todos los ítems de motivación, este clúster presenta la media más alta con respecto al resto de grupos. Su principal motivación también es la "Relajación" y el ítem que menos promedio muestra en el grupo es el "Vínculo con actividades comerciales". Los demás ítems "Conciencia por la salud", "Realizar terapia física", "Realizar una actividad social", "Recreación", "Mejora de la calidad de vida", "Ser una actividad asequible económicamente", "Relajación", "Recomendación de otros", "Ser una actividad atractiva", "Meditación", entre otros son valorados por encima de la media total.

Está conformado en su mayor parte por personas con ingresos medios familiares de 601 a 1200 euros $(45,8 \%)$, siendo el mayor porcentaje de personas con dichos ingresos con respecto a los cuatro grupos. El segundo mayor porcentaje son las personas con ingresos familiares de 1201 a 1800 euros (22\%). Casi las dos terceras partes de este clúster sí ha realizado turismo de bienestar $(64,3 \%)$ y este porcentaje se encuentra entre los más altos. También la gran mayoría $(44,8 \%)$ está familiarizado con el término "bienestar" y "bastante" familiarizados con la provincia de Granada como destino de este tipo de turismo (42\%) siendo también el porcentaje más alto de los cuatro grupos. Por otro lado para sus integrantes es probable $(37,4 \%)$ o muy probable $(42,6 \%)$ que Granada esté entre sus opciones de vacaciones en un futuro cercano y también que lo sea en algún momento de su vida $(38,5 \%)$ y $(49,7 \%)$. Así mismo entre sus opciones es muy probable que se encuentren establecimientos centrados en turismo de bienestar en sus futuras vacaciones $(37,8 \%)$.

Grupo 3: El tercer grupo representa el $27,8 \%$ de la muestra (112 integrantes) y constituye el segundo segmento más numeroso. Se trata de un grupo formado por "Oportunistas" al valorar en mayor medida aspectos relacionados con el disfrute, el contacto con otros, la accesibilidad de las actividades entre otros aspectos. De los cuatro grupos el ítem con mayor media es "Actividad que no requiere esfuerzo" y dentro del grupo el ítem que más valoran es la "Relajación" y el de menor media es el de "Meditación". Es el clúster que aglutina en su mayoría a las personas con ingresos familiares altos de más de 1801 euros (31\%) tanto dentro del clúster como con respecto al resto. A continuación le siguen las personas con ingresos familiares de 601 a 1200 euros $(27,6 \%)$.

La mayoría de este clúster también ha realizado turismo de bienestar $(64,3 \%)$ pero aproximadamente la mitad $(41,4 \%)$ no está familiarizado con el término o no conoce su significado. También se consideran neutrales en su mayoría $(37,7 \%)$ con respecto a la familiaridad con la provincia de Granada como destino turístico de bienestar. Este grupo, junto con el segundo, es el que presenta un mayor porcentaje de individuos que han realizado este tipo de turismo. También registra una gran mayoría con una calificación de "probable" con respecto a la intención de ir a Granada para vacaciones en los próximos tres años $(31,7 \%)$ o de visitar establecimientos centrados en turismo de bienestar (50\%) y muy probable ir a Granada en algún momento de su vida (39,3\%).

Grupo 4: La cuarta agrupación extraída representa el 12,9\% de la muestra (52 integrantes). Se trata de un grupo de "Desmotivados" siendo el que menor puntuación media manifiesta en casi todos los ítems. El valor promedio más bajo se produce en el item "Vínculo con actividades comerciales" tanto dentro del grupo como con respecto a los otros tres y la mayor puntuación es para la "Relajación" como también ocurre en los demás grupos. En general valoran por debajo de la media total ítems como "Realizar terapia mental", "Ser una actividad atractiva", "Disfrute de la naturaleza", "Meditación", "Conciencia por la salud", "Realizar terapia física" y "Realizar una actividad social".

Es el que mayor porcentaje de personas con sueldo de hasta 600 euros tiene de los 4 grupos (24,4\%). Dentro del grupo también obtienen mayoría las personas con ingresos familiares de 601 a 1200 euros (29,3\%) y más de 1801 euros (29,3\%). El 63,5\% de los integrantes de este clúster sí ha realizado turismo de bienestar frente a un $36,5 \%$ que no lo han realizado, sin embargo, el $20,7 \%$ de los integrantes se encuentra nada familiarizado con el término "bienestar" y con la provincia de Granada como destino (29,6\%). También la tercera parte aproximadamente (27,3\%) expresa que es improbable visitar Granada en los próximos tres años como opción de vacaciones, pero que sí es muy probable que la visiten en algún momento de su vida $(42,3 \%)$ y probablemente asistirán a establecimientos centrados en turismo bienestar en sus próximas vacaciones (36,5\%).

\subsection{Determinantes de la predisposición a visitar un turismo de bienestar}

Una vez revisados y cumplidos los supuestos de partida para realizar el modelo de regresión múltiple se procede a contrastar las hipótesis $\mathrm{H} 1, \mathrm{H} 2, \mathrm{H} 3$ y $\mathrm{H} 4$.

La estimación del modelo y los supuestos de partida se basan en los resultados del modelo de regresión tras la eliminación de valores atípicos, extremos u outliers en un primer paso. Una vez eliminados cuatro valores atípicos no se aprecia ningún otro caso con un valor del residuo tipificado mayor que 3.

Para encontrar los mejores estimadores de la regresión (Hair, Gómez-Suárez, Cano \& Prentice, 1999) se optó por utilizar el método de eliminación regresiva (hacia atrás). Los coeficientes de 
regresión y otros estadísticos asociados a cada variable explicativa (familiaridad, motivación, imagen cognitiva e imagen afectiva) de la intención comportamental aparecen recogidos en la Tabla 6. Los resultados demuestran que la intención de visita de un destino turístico de bienestar puede ser explicada por las variables independientes familiaridad, motivación e imagen cognitiva y afectiva. Además las relaciones resultaron en el sentido esperado.
Derivado de todo esto se puede decir que, para los turistas de bienestar, la familiaridad, la motivación y la imagen cognitiva y afectiva influyen positivamente sobre la intención de visita de un destino de turismo de bienestar. Por tanto, existe evidencia empírica para aceptar la veracidad de lo sostenido por las hipótesis $\mathrm{H} 1, \mathrm{H} 2, \mathrm{H} 3$ y H4, tal y como la teoría anticipa.

Tabla 6 - Coeficientes de Regresión - Intención (dep.)

\begin{tabular}{|l|c|c|c|c|c|}
\hline \multicolumn{1}{|c|}{ Variable Independiente } & B & Error típico & $\boldsymbol{\beta}$ estand. & $\mathbf{t}$ & Sign. \\
\hline (Constante) & 0,044 & 0,292 & ---- & 0,150 & 0,881 \\
\hline Familiaridad & 0,065 & 0,031 & 0,089 & 2,084 & 0,038 \\
\hline Motivación & 0,127 & 0,060 & 0,091 & 2,116 & 0,035 \\
\hline Imagen Cognitiva & 0,557 & 0,074 & 0,384 & 7,562 & 0,000 \\
\hline Imagen Afectiva & 0,262 & 0,063 & 0,202 & 4,177 & 0,000 \\
\hline R2=0,345, g.l. 4; F=52,179; sign.>0,000 & & & & & \\
\hline
\end{tabular}

El menor coeficiente se encuentra en la variable familiaridad con un peso estandarizado $(\beta)$ de 0,089 . Por su parte la motivación con un 0,091 de la intención, la imagen afectiva el 0,202 y la imagen cognitiva es la que mayor peso obtiene $(\beta=0,384)$.

El coeficiente de determinación $\left(R^{2}\right)$ muestra que para este conjunto de datos el modelo en su conjunto tiene una capacidad explicativa del $34,5 \%$ de la variabilidad de intención de visita.

\section{Conclusiones finales, limitaciones y futuras líneas de investigación}

\subsection{Discusión de resultados e implicaciones para la gestión}

El presente artículo se ha encargado de analizar este segmento de mercado emergente y con carácter exploratorio se plantearon varios objetivos. La consecución de dichos objetivos pasó por el diseño de un cuestionario web autoadministrado a un grupo de turistas actuales y potenciales de este tipo de turismo.

Con respecto a las motivaciones subyacentes al realizar turismo de bienestar identificamos cuatro grupos diferenciados de turistas de bienestar. El factor motivacional común a todos los grupos es la relajación que coincide con los resultados encontrados en el trabajo de Konu y Laukkanen (2009) y Voigt, Brown y Howat (2011) quienes investigaron la influencia de las motivaciones en la intención de realizar un viaje de bienestar y encontraron este ítem en común en todos los grupos.

Un primer grupo es denominado los "Desinteresados" que representan el $23,8 \%$ de la muestra. La mayoría son personas con ingresos familiares de 1201 a 1800 euros (37\%), algo más de la mitad no ha realizado turismo de bienestar $(54,2 \%)$, se encuentran poco familiarizados con el término o destinos relacionados $(40,6 \%)$ y no manifiestan una intención próxima a visitar destinos o realizar este tipo de turismo (36,5\%). El segundo grupo corresponde a los "Motivados" que recoge a un tercio de la muestra donde casi dos tercios del total sí han realizado turismo de bienestar y se encuentran interesados por todos los beneficios que pueden obtener al realizarlo. Están en su mayoría familiarizados con el término y destinos ( $44,8 \%$ y $32,9 \%$ respectivamente) y presentan el porcentaje más alto de intención a visitar y realizar este tipo de turismo a corto plazo $(38,5 \%)$. El tercer grupo son los denominados "Oportunistas" pues valoran en mayor medida aspectos utilitarios derivados del turismo de bienestar. Es el grupo con un gran porcentaje de ingresos familiares elevados $(31 \%)$, casi dos tercios sí han realizado turismo de bienestar, pero no están familiarizados con el término $(41,4 \%)$. El cuarto grupo se denomina los "Desmotivados" al manifestar bajas puntuaciones en casi todas las motivaciones, son personas en su mayoría con menores ingresos familiares que los otros grupos con un sueldo de hasta 600 euros entre un cuarto aproximadamente de integrantes, que sí han realizado turismo de bienestar pero se encuentran poco familiarizados con el término (39.5\%) y manifiestan neutralidad en cuanto a la intención de visitar destinos como Granada en un futuro cercano.

El modelo de regresión obtenido revela que un 34,5\% de la varianza de la intención de realizar turismo de bienestar o visitar destinos de este tipo de turismo se debe a las variaciones de las variables motivación, imagen cognitiva y afectiva y familiaridad. La relación de la imagen (cognitiva y afectiva) con la intención se ha encontrado en trabajos como los de Baloglu y MacCleary (1999), Crompton (1979) y Hunt (1975) donde sólo los destinos con una imagen fuerte se consideran importantes en el proceso de selección y se mantienen entre las posibles opciones a seleccionar.

Centrando la atención en la motivación se encuentran resultados similares en los trabajos de Uysal y Hagan (1993) y Weaver, McCleary, Lapisto y Damonte (1994) que encuentran que la motivación juega un papel importante en el proceso de elección de un destino turístico.

La baja influencia de la familiaridad en la intención puede ser debido a que esta variable ejerce un efecto directo en la intención, pero también indirecto a través de la imagen como se ha comprobado en el estudio de Hammitt et al. (2006).

Los administradores de empresas y destinos turísticos deberían crear mensajes de marketing basados en los beneficios que los posibles turistas de bienestar están buscando, las necesidades que desean ver satisfechas y armar paquetes y programas a la medida de esas expectativas.

Las acciones de marketing deben ir dirigidas especialmente a mujeres y en general a personas con estudios universitarios y de posgrado porque son las que en mayor medida realizan turismo de 
bienestar. Además atendiendo a las motivaciones se deben crear paquetes diferenciados para los cuatro grupos identificados, pero que también manifiesten claramente la principal motivación de estos turistas que es la relajación. La comunicación destinada al primer grupo de "desinteresados" debe enfatizar la mejora en la calidad de vida y el aspecto físico pero sin implicar gran esfuerzo. Para el segundo grupo de "motivados", al conocer ya este tipo de turismo y lo han practicado alguna vez los mensajes deben acentuar diferentes beneficios relacionados con la recreación, la interacción social y la mejora de la calidad de vida.

Con respecto al tercer grupo de "oportunistas", al valorar aspectos utilitarios se debería hacer hincapié en actividades atractivas y beneficios altos o mínimo esfuerzo y mejora del bienestar. Además de mensajes que incrementen su familiaridad con el término para que se pueden ver más proclives a realizarlo en el futuro. Es un grupo con gran potencialidad al declarar mayores ingresos y con una probabilidad alta de asistir a destinos relacionados con turismo de bienestar.

Por último para el grupo de los "desmotivados" se deberían diseñar acciones de comunicación que resalten la relación costebeneficio al tratarse de personas que sí han realizado este tipo de turismo pero no conocen bien los beneficios que conlleva y por tanto no se encuentran lo suficientemente motivados para realizarlo próximamente.

\subsection{Limitaciones y futuras líneas de investigación}

Aunque el uso de muestras de estudiantes para la investigación experimental ha sido criticado muchas veces, desde el punto de vista de investigación en turismo, los estudiantes universitarios son un segmento poco atendido que ha atraído la atención de muchos autores recientemente debido a que se encuentra en crecimiento y se ha convertido en un segmento lucrativo para el mercado de viajes (Gallarza \& Saura, 2006).

Si bien, las respuestas de estudiantes tienden a ser más homogéneas (Peterson, 2001) y con tales muestras resulta más fácil refutar la teoría (Calder, Phillips \& Tybout, 1981), los resultados deberían ser extrapolados con precaución (O'Neil, 2001). La motivación de los estudiantes puede ser diferente de la que tienen personas mayores de 30 años para las cuales la salud y el bienestar es un asunto más prioritario y en la mayoría de la literatura encontramos que este tipo de actividades de bienestar es realizada en mayor medida por este segmento de edad. Dada la escasez de investigaciones relacionadas con las motivaciones, imagen y familiaridad de los turistas de bienestar, el presente estudio sirve como un estudio preliminar dentro de una línea más amplia de investigación que debe realizarse con poblaciones de adultos mayores.

Adicionalmente el término "bienestar" como concepto y como industria no está totalmente desarrollado. Todavía es considerado una extensión de la industria de la belleza por muchos, de la salud por otros, de fitness y culto al cuerpo por unos pocos e incluso de cuidados post operativos por algunos. Por este motivo la industria todavía tiene que formarse como una entidad independiente y el estudio presentado es únicamente de carácter exploratorio.
Como futura línea de investigación se propone analizar a diferentes audiencias objetivo o tipos de usuario; lo que permitiría verificar el valor general o validez externa de los resultados obtenidos. También se debe realizar un nuevo análisis de las escalas usando una muestra más representativa de la población en términos de edad, ocupación e ingresos. De esta manera se lograría una visión más amplia del fenómeno de bienestar que se pueda aplicar en diferentes lugares.

En segundo lugar como el término bienestar es un concepto complejo donde intervienen muchos factores, resulta fundamental tener en cuenta una serie de variables que no se han analizado en este trabajo tales como la satisfacción, formación de lealtad, imagen de marca y co-creación. En futuras líneas de investigación sería interesante desarrollar un modelo de comportamiento que incluyera estas variables. Sin embargo el sector de bienestar es un sector emergente y cada aspecto de esta nueva industria puede ser investigado creando muchas líneas de investigación futuras posibles.

\section{Referencias}

Alegre, J., \& Cladera M. (2009). Analysing the effect of satisfaction and previous visits on tourist intentions to return. European Journal of Marketing, 43(5/6), 670-685. doi: 10.1108/03090560910946990.

Baloglu, S. (2000). A path analytic model of visitation intention involving information sources, socio-psychological motivations, and destination image. Journal of Travel \& Tourism Marketing, 8(3), 81-90. doi: 10.1300/J073v08n03_05.

Baloglu, S., \& Mangaloglu, M. (2001). Tourism destination images of Turkey, Egypt, Greece, and Italy as perceived by US-based tour operators and travel agents. Tourism Management, 22(1), 1-9.

Baloglu, S., \& McCleary, K. W. (1999). A model of destination image formation. Annals of Tourism Research,26(4), 868-897. doi: 10.1016/S0160-7383(99)00030-4.

Beerli, A., \& Martín, J.D. (2004). Como influyen las fuentes de información en la imagen percibida de los destinos turísticos, Revista española de investigación de marketing, 8(2), 2004, 7-34.

Bettman, J. R. (1979). Memory factors in consumer choice: A review. Journal of Marketing (Pre-1986), 43(2), 37-37.

Bigné, J. E., Sánchez, M. I., \& Sánchez, J. (2001). Tourism image, evaluation variables and after purchase behaviour: Inter-relationship. Tourism Management, 22(6), 607-616. doi: 10.1016/S0261-5177(01)00035-8

Bojanic, D. C. (1991). The use of advertising in managing destination image. Tourism Management, 12(4), 352-355. doi: 10.1016/02615177(91)90047-W.

Bornstein, R. F., \& D'Agostino, P. R. (1992). Stimulus recognition and the mere exposure effect. Journal of Personality and Social Psychology, 63(4), 545-552. doi:10.1037/0022-3514.63.4.545.

Byon, K. K., \& Zhang, J. J. (2010). “Development of a scale measuring destination image". Marketing Intelligence \& Planning, 28(4), 508-532. doi:10.1108/02634501011053595.

Calder, B.J., Phillips L.W., \& Tybout A.M., (1981) Designing research for application, Journal of Consumer Research, 8(2), 197-207.

Cameron, F., Cornish, C., \& Nelson, W. (2006). A new methodology for segmenting consumers for financial services. Journal of Financial Services Marketing, 10(3), 260-271.

Chen, C., \& Tsai, D. (2007). How destination image and evaluative factors affect behavioral intentions? Tourism Management, 28(4), 1115-1122. doi: 10.1016/j.tourman.2006.07.007. 
Chen, J. S., Prebensen, N., \& Huan, T. C. (2008). Determining the motivation of wellness travelers. Anatolia, 19(1), 103-115. doi: 10.1080/13032917.2008.9687056.

Chen, K. (1997). The effect of satisfaction and familiarity on intention: Multiple-destination context.Journal of International Consumer Marketing, 10(1), 159-173.

Chica, J., \& Frías, D. M. (2000). “Regresión Lineal”, en T. Luque (coord.), Técnicas de análisis de datos en investigación de mercados, (pp.247-280). Madrid: Ed. Pirámide.

Crompton, J. L. (1979). Motivations for pleasure vacation. Annals of Tourism Research, 6(4), 408-424. doi: 10.1016/0160-7383(79)90004-5.

Dolnicar, S. (2002). A review of data-driven market segmentation in tourism, Journal of Travel \& Tourism Marketing, 12(1), 1-22. doi:10.1300/J073v12n01_01.

Durkin, M. (2004). In Search of the Internet-Banking Customer: Exploring the Use of Decision Style. The International Journal of Bank Marketing, 22(6/7), 484-503.

Echtner, C. M., \& Ritchie, J. R. B. (1993). The measurement of destination image: An empirical assessment. Journal of Travel Research, 31(4), 3-3.

Frochot, I., \& Morrison, A. M. (2000). Benefit segmentation: A review of its applications to travel and tourism research, Journal of Travel \& Tourism Marketing, 9(4), 21-45.

Gallarza, M. G., \& Saura, I. G. (2006). Value dimensions, perceived value, satisfaction and loyalty: An investigation of university students' travel behaviour. Tourism Management, 27(3), 437-452. doi:10.1016/j.tourman.2004.12.002.

Garland, R. (2005). Segmenting Retail Banking Customers. Journal of Financial Services Marketing, 10(2), 179-191.

Gartner, W. C. (1993). Image formation process. Journal of Travel \& Tourism Marketing, 2(2-3), 191-216. doi: 10.1300/J073v02n02_12.

Hair, J. F., Gómez-Suárez, M., Cano, D., \& Prentice, E. (1999). Análisis multivariante. Madrid: Prentice Hall Iberia.

Hammitt, W. E., Backlund, E. A., \& Bixler, R. D. (2006). Place bonding for recreation places: Conceptual and empirical development. Leisure Studies, 25(1), 17-41. doi: 10.1080/02614360500098100.

Hanlan, J., \& Kelly, S. (2005). Image formation, information sources and an iconic Australian tourist destination. Journal of Vacation Marketing, 11(2), 163-177.

Henn, M. R., Lopes, P., Goncalves, J. M., \& Fraiz, J. A. (2008). Turismo termal: cambios conceptuales y mercadológicos de los balnearios en España. Revista Turismo Visao E Acao, 10, 415-434.

Hunt, J. D. (1975). Image as a factor in tourism development. Journal of Travel Research, 13(3), 1-7. doi: 10.1177/004728757501300301.

Jang, S., \& Feng, R. (2007). T.emporal destination revisit intention: The effects of novelty seeking and satisfaction. Tourism Management, 28(2), 580-590.

Jeong, C. (2009). Effects of exposure time to travel information sources on familiarity, destination image and intention to visit. Recuperado Agosto 28, 2012 de: http://search.proquest.com/docview/880896867.

Jeong, C., Holland, S., Jun, S. H., \& Gibson, H. (2012). Enhancing destination image through travel website Information. International Journal of Tourism Research, 27(8), 16-27.

Johnson, E. J., \& Russo, E, J. (1984). Product familiarity and learning new information. Journal of Consumer Research (Pre-1986), 11(1), 542-542.

Karjalouto, H., Mattila, M., \& Pento, T. (2002). Factors underlying attitude formation toward online banking in Finland, International Journal of Bank Marketing, 20 (6), 261- 272.

Kaynak, E., \& Harcar, T. D. (2005). American Consumer's Attitudes Towards Commercial Banks. A Comparison of Local and National Bank Customers by
Use of Geodemographic Segmentation. The International Journal of Bank Marketing, 23(1), 73-89.

Kent, R. J., \& Allen, C. T. (1994). Competitive interference effects in consumer memory for advertising: The role of brand familiarity. Journal of Marketing, 58(3), 97-97.

Konu, H., \& Laukkanen, T. (2009). Roles of motivation factors in predicting tourists'intentions to make wellbeing holidays-a Finnish case. ANZMAC 2009 - Sustainable Management and Marketing Proceedings. Recuperado Julio 20, 2012 de: http://www.duplication.net.au/ANZMAC09/papers/ANZMAC2009376.pdf.

Lam, T., \& Hsu, C. H. C. (2006). Predicting behavioral intention of choosing a travel destination. Tourism Management, 27(4), 589-599. doi: 10.1016/j.tourman.2005.02.003

Larrubia-Vargas, R., \& Luque-Gil, A. M. (2002). Las estaciones termales en Andalucía: de la explotación tradicional a la configuración de un nuevo producto turístico integral. Cuadernos de Turismo, 10, 101-122.

Lee, E. J., Kwon, K. N., \& Schumann, D. W. (2005). Segmenting the nonadopters category in the diffusion of Internet banking, The International Journal of Bank Marketing, 23 (4/5), 414-437.

Letho, X.Y., Brown, S., Chen, Y., \& Morrison, A.M. (2006), "Yoga tourism as a niche within the wellness tourism market", Tourism Recreation Research, 31 (1) 25-35.

Loh, C. P. A. (2014). Health tourism on the rise? Evidence from the balance of payments statistics. European Journal of Health Economics, 15(7), 759766. http://doi.org/10.1007/s10198-013-0521-0.

Luque -Martínez, T. (2000).Técnicas de análisis de datos en investigación de mercados. Madrid: Ed. Pirámide.

Luque-Martínez, T., Garcia, S. del B., Ibañez Zapata, J. A., \& Rodriguez Molina, M. A. (2004). "La modelización de la imagen de Granada desde la perspectiva de los líderes de opinión externos". En XVI Encuentro de Profesores Universitarios de Marketing. Alicante, España.

Machauer, A., \& Morgner, S. (2001). Segmentation of bank customers by expected benefits and attitudes. International Journal of Bank Marketing, 19 (1), 6-17.

Mansfeld, Y. (1992). From motivation to actual travel. Annals of Tourism Research, 19(3), 399-419. doi: 10.1016/0160-7383(92)90127-B.

Marks, L. J., \& Olson, J. C. (1981). Toward a cognitive structure conceptualization of product familiarity. Advances in consumer research, 8 (1), 145-150.

Martín, H. S., \& Bosque, I. A. R. (2008). Exploring the cognitive-affective nature of destination image and the role of psychological factors in its formation. Tourism Management, 29, 263-277.

Martínez, J. A. (2014). “A methodological proposal to evaluate a city image through associative maps". Cuadernos de Gestión, 14(1), 75-96. http://doi.org/10.5295/cdg.120361jm.

Mechinda P., Serirat S., \& Gulid N. (2009). An examination of tourists' attitudinal and behavioral loyalty: Comparison between domestic and international tourists. Journal of Vacation Marketing, 15(2), 129-148.

Milman, A., \& Pizam, A. (1995). The role of awareness and familiarity with a destination: The central florida case. Journal of Travel Research, 33(3), 21-27. doi: 10.1177/004728759503300304.

Moreno Gil, S., Beerli Palacio, A., \& Ledesma, J. de L. (2012). “Entender la imagen de un destino turístico: Factores que la integran y la influencia de las motivaciones". Criterio Libre, (16), 115-142.

Morgan, D. (2010). "The growth of medical tourism". Recuperado Marzo, 9, 2015, de http://www.oecdobserver.org/news/fullstory.php/aid/3342/The_growth _of_medical_tourism.html. 
Mueller, H., \& Kaufmann, E. L. (2001). Wellness tourism: Market analysis of a special health tourism segment and implications for the hotel industry. Journal of Vacation Marketing, 7(1), 5-17. http://doi.org/10.1177/135676670100700101.

Nunnally, J.C. (1978).Psychometric Theory, 2oed. New York.: McGraw- Hill,

O'Neil, D. (2001). "Analysis of Internet users level of online privacy concerns", Social Science Computer Review, 19, 17-31.

Pearce, P. (1982). The Social Psychology of Tourist Behaviour. Oxford: Pergamon.

Pearcy, D. H., \& Lester, J. (2012). Capitalizing on emerging tourism trends: An exploratory examination of Jamaica's wellness tourism sector within an innovation systems framework. International Journal of Business, Marketing, and Decision Sciences, 5(2), 121-137.

Peterson, P.W. (2001). The Debate About online Learning: Key Issues for Writing Teachers, Computers and Composition, 40, 57-70.

Phau, I., Shanka, T., \& Dhayan, N. (2010). Destination image and choice intention of university student travellers to Mauritius. International Journal of Contemporary Hospitality Management, 22(5), 758-764. http://doi.org/10.1108/09596111011053846.

Pons, J. (2007). Web 2.0 y Travel 2.0, in Resumen tecnologías sociales aplicadas al turismo. Recuperado Junio,13, 2013 de: www.hosteltur.com.

Puczkó, L., \& Bachvarov, M. (2006). Spa, bath, thermae: What's behind the labels? Tourism Recreation Research, 31(1), 83-91.

Rajshekhar G. J., \& Dion, P. (1999). A Life Cycle Segmentation Approach to Marketing Financial Products and Services. The Service Industries Journal, 19(3), 74-96.

Ravichandran, S., \& Suresh, S. (2010). Using Wellness Services to Position and Promote Brand India. International Journal of Hospitality \& Tourism Administration, 11(2), 200-217. http://doi.org/10.1080/15256481003732873 .

Reddy, S. G., York, V. K., \& Brannon, L. A. (2010). Travel for treatment: Students' perspective on medical tourism. International Journal of Tourism Research, 12(5), 510-522. http://doi.org/10.1002/jtr.769.

Schwaiger, M., \& Locarek-Junge, H. (1998). Realizing customer retention potentials by electronic banking, Electronic markets, 8(4), 23-26.

Smith, M., \& Puczkó, L. (2009). Health and wellness tourism. Oxford: Butterworth-Heinemann.

Soper, S. (2002). The evolution of segmentation methods in financial services: Where next?. Journal of Financial Services Marketing, 7(1), 67-74.

Universidad de Granada (2011). Estudio de egresados de la Universidad de Granada. La inserción laboral en el Campus de Excelencia Internacional. Universidad de Granada. Recuperado Junio,16, 2012 de: http://marketing.ugr.es/encuesta/stats14/frameset.htm.

Uysal, M., \& Hagan L. A. R. (1993). Motivation of Pleasure Travel and Tourism. In VNR's Encyclopedia of Hospitality and Tourism, (pp.798-810). New York: Van Nostrand Reinhold.

Vogt, C. A.,\& Fesenmaier, D. R. (1998). Expanding the functional information search model. Annals of Tourism Research, 25(3), 551-578. doi: 10.1016/S0160-7383(98)00010-3.

Voigt C., Brown G., \& Howat G. (2011). Wellness tourists: In search of transformation. Tourism Review of AIEST - International Association of Scientific Experts in Tourism, 66(1/2), 16-30. doi: 10.1108/16605371111127206.

Weaver, P. A., McCleary, K. W., Lapisto, L., \& Damonte, L. T. (1994). The relationship of destination selection attributes to psychological, behavioral and demographic variables. Journal of Hospitality \& Leisure Marketing, 2(2), 93-109. doi: 10.1300/J150v02n02_07.
Winsted, K. F. (1997). The service experience in two cultures: A behavioral perspective. Journal of Retailing, 73(3), 337-360. http://doi.org/10.1016/S0022-4359(97)90022-1.

Yoon, Y., \& Uysal, M. (2005). An examination of the effects of motivation and satisfaction on destination loyalty: A structural model. Tourism Management, 26(1), 45-56. doi: 10.1016/j.tourman.2003.08.016.

Zajonc, R. B. (1968). Attitudinal effects of mere exposure. Journal of Personality and Social Psychology, 9(2), 1-27. doi: 10.1037/h0025848.

Recibido: 21.10.2015

Reenviado: 17.06 .2015

Aceptado: 18.06.2016 